\title{
Robotic Process Automation in the Automotive Industry - Lessons Learned from an Exploratory Case Study
}

\author{
Judith Wewerka $\bigotimes^{1,2[0000-0002-4809-2480]}$ and \\ Manfred Reichert ${ }^{1[0000-0003-2536-4153]}$ \\ 1 Institute of Databases and Information Systems, Ulm University, Germany \\ \{judith.wewerka, manfred.reichert\}@uni-ulm.de \\ 2 Research and Development, BMW Group, Germany
}

\begin{abstract}
Robotic Process Automation (RPA) is the rule-based automation of business processes by software bots mimicking human interactions. The aims of this paper are to provide insights into three RPA use cases from the automotive domain as well as to derive the main challenges to be tackled when introducing RPA in this domain. By means of an exploratory case study, the three use cases are selected from real RPA projects. A systematic method for analyzing the cases is applied. The results are structured along the stages of the lifecycle model of software development. We provide information on every lifecycle stage and discuss the respective lessons learned. In detail, we derive five challenges that should be tackled for any successful RPA implementation in the automotive domain: (1) identifying the right process to automate, (2) understanding the factors influencing user acceptance, (3) explaining RPA to the users, (4) designing human bot interaction, and (5) providing software development guidelines for RPA implementation.
\end{abstract}

Keywords: Robotic Process Automation, RPA, Exploratory Case Study, Use Case, Automotive Industry.

\section{Introduction}

To stay competitive in their market, companies need to organize their business processes in an efficient and cost-effective manner [11]. They, therefore, demand for an increasing degree of process automation. A promising approach is provided by Robotic Process Automation (RPA), which aims to automate business processes or parts of them by software robots (bots for short) mimicking human interactions. Thus, an increasing number of companies have been running RPA initiatives [2].

Scientifically, RPA has been investigated in various directions, e.g., systematic literature reviews [6,17], case studies in areas like shared services [10,22] and telecommunications $[16,23]$, methods fostering RPA implementation $[4,7]$, and attempts to quantify RPA effects [20]. However, there exists little research on introducing RPA in the automotive industry. Consequently, lessons learned 
resulting from RPA application in this domain have not been extensively reported in literature yet. This raises the question whether and-if yes-how RPA projects in automotive industry can be successfully completed.

The purpose of this paper is to gain a better understanding of RPA projects. Moreover, we want to highlight the challenges that need to be tackled to enable a successful RPA implementation. We conduct an exploratory case study in the automotive industry to find out what is happening, to gain novel insights into real world projects, and to generate ideas for new research [21].

The remainder of the paper is organized as follows: Section 2 summarizes existing RPA case studies and the lessons learned in their context. Section 3 describes the method we applied for this case study research. The results are presented in Section 4, followed by a cross-case analysis and a systematic derivation of the challenges in automotive industry in Section 5.

\section{Related Work}

We summarize results from existing RPA case studies with a focus on the lessons learned for a successful RPA implementation. To the best of our knowledge, no case study exists that has been conducted in the automotive domain. Therefore, we focus on giving an overview of RPA case studies in general. These lessons learned are used in Section 5 to classify our results.

In [8], RPA in business process outsourcing is introduced with the goal to create and validate premium advice notes. Overall, 14 processes are automated with 120.000 cases per month resulting in cost savings of $30 \%$. Eight lessons learned are emphasized: (1) RPA needs a sponsor, (2) a culture of business innovation and technology accelerates RPA adoption, (3) RPA should sit in the business, (4) processes should be standardized and stabilized before automation, (5) RPA must comply with enterprise architecture policies, (6) internal RPA capability should be built, (7) bots should be multi-skilled, and (8) internal communication is highly important for RPA projects.

The RPA journey of an energy supplier is presented in [9]. One process is automated to resolve infeasible customer meter readings. In total, 25 processes are automated with 1 million cases per month. The lessons learned that are reported in [8] are picked up and expanded with further aspects: the composition of the RPA teams should evolve over time, continuous prototyping becomes necessary to expand RPA to new business contexts, RPA should complement enterprise information systems, and components should be reused to scale quickly and to reduce development costs.

The RPA journey of a shared service company automating the generation of the financial close is described in [10]. Overall, 44 processes are automated saving 45 Full Time Equivalents (FTEs). The lessons learned are to pick the right RPA approach (i.e., to differentiate between screen automation and process automation), to redesign work prior to implementation, and to prepare the employees. [22] presents another RPA journey in shared service highlighting sub-processes that may be subject to RPA, e.g., copy data from an Excel sheet to a human 
resource management system. Finally, [22] emphasizes that RPA benefits are immense, but results (e.g., faster processes or FTE savings) cannot be always guaranteed.

An RPA journey of a telecommunication company is presented in [16]. Two processes are discussed, i.e., bundle support tools for the field service technician on one hand and proactive problem solving on the other. During the RPA journey, 800 FTEs are saved. As lessons learned, RPA should be designed and implemented in an agile way, RPA impact on staff members should be carefully managed, and technical and organizational interrelations should be investigated from the beginning. Another RPA journey in telecommunications is described in [23]: 15 processes are automated with around 500.000 cases per month. RPA is considered as one tool working hand in hand with process elimination, process improvement, and other business process tools. As the two main lessons learned, bots require more explicit instructions than humans and some risks need to be taken to successfully introduce RPA.

How to automate the generation of a payment receipt with a business process outsourcing provider is described in [1]. The as-is and to-be processes are described, and it is shown how RPA increases productivity, e.g., the group using the RPA bot could handle $21 \%$ more cases than the one without bot. Another RPA use case in the same domain is presented in [5]-the automated processes update employee payment details and create employment relationships. The lessons learned include preparing the IT department and developing RPA capabilities, addressing fears about losing jobs, selecting processes carefully, and measuring process improvements.

In [3], RPA is applied to digital forensics in two use cases: keyword search on forensic platforms and import evidence files to a forensic platform. As main lesson learned, special forensic software cannot be properly automated with available RPA software solutions.

The case study presented in [12] emphasizes the benefits of RPA for mastering data management. Companies are accompanied to show the qualitative benefits achieved with RPA, including improved data quality, reduced human errors, increased productivity, and decreased costs. As particular lesson learned it is mentioned to overcome the lack of understanding RPA technology.

In [24], an RPA system supporting corporate service providers is presented. Both the as-is and the to-be processes are described, and RPA is evaluated quantitatively. Concrete numbers to productivity improvements (over 1,000\%) are provided and managerial impacts are derived. No lessons learned are given.

Finally, [18] investigates RPA use cases in procurement to explore practical implications and the impact on the procurement functions. The use cases are further analyzed regarding challenges during RPA implementation, e.g., employees fear to change their working habits, or the procurement function needs to be mature. Details on the concrete cases are missing. 


\section{Methodology}

This section presents the method we applied to conduct the RPA case study in the automotive domain. A case study in software engineering is "an empirical enquiry that draws on multiple sources of evidence to investigate one instance (or a small number of instances) of a contemporary software engineering phenomenon within its real-life context" [14] to improve the software engineering process and the resulting product, i.e., the RPA bot in the context of this work [14]. We combine the methodologies presented in [14], [21], and [25] and obtain the following method: first, the case study is designed, research questions are derived, and use cases are selected (cf. Section 3.1). Second, the data collection method is defined (cf. Section 3.2). Third, the data analysis method is selected (cf. Section 3.3).

\subsection{Case Study Design}

Introducing RPA in the automotive domain has been little studied in the literature and, hence, concrete RPA applications, i.e., use cases, are still rare. Existing case studies, e.g., [1], focus on the benefits, risks, and results of RPA. The aim of the study presented in this paper is to explore RPA use cases in the automotive industry along the software lifecycle model [13]. Furthermore, the challenges to be tackled for each lifecycle stage are derived $[4,7]$ :

- The Analysis Stage focuses on understanding and analyzing the as-is process, which is the candidate to be automated with RPA.

- The Product Design Stage defines the to-be process that shall be executed by the bot.

- The Coding and Testing Stage implements the RPA bot according to the design defined in the previous stage. The bot is tested to determine whether it behaves correctly.

- The Operation Stage deploys, maintains, and operates the RPA bot. Moreover, it measures its performance.

Research Question Definition. In [20], we discovered that RPA projects in the automotive industry do not always achieve the positive benefits promised in the literature. Therefore, our general research question is "What challenges are raised in each lifecycle stage?". In detail, different aspects in each stage are questioned to answer the general research question. For the analysis stage we consider the following aspects: (1) How does the as-is process look like? (2) What problems exist with the as-is process?, and (3) What are the goals of the RPA project? The product design stage, in turn, addresses the two aspects: (1) How does the to-be process look like? and (2) Is the process standardized, i.e., can the number of process variants be kept small? For the coding and testing stage we consider the following aspects: (1) How long does coding and testing of the RPA bot take? and (2) What problems arise in this stage? Finally, the operation stage addresses four aspects, namely: (1) When is the bot released? (2) How is the bot 
communicated to the users? (3) How much time is needed for maintenance?, and (4) What are the lessons learned in the RPA project?

Case Selection. In order to select appropriate cases to identify general challenges of RPA implementation in the automotive domain, we consider the following criteria [21]: availability of information, variation of cases, and repeatability. In this context, it is noteworthy that one of the authors deals with the selection of processes in RPA projects in automotive engineering. Over a period of one and a half years, she decided in 42 cases whether or not the suggested process should be automated with RPA (cf. Figure 1). 31 of them were rejected as they were unsuitable for RPA (19), did not save enough FTEs (8), or for other reasons (4). Hence, 11 processes were automated with RPA, of which two each failed during coding and testing, respectively. Two processes are still in the testing stage, five were successfully completed. Thereof two are retired and three are actively used. Information was available for all 42 processes as one of the author is part of the RPA team. To ensure the variation of cases, we want to explore one process that failed after testing, one successfully completed, and one being active. Finally, the repeatability criteria results in the selection of the processes called Process 1 (Ordering), Process 2 (Construction Report Creation), and Process 3 (Report Generation).

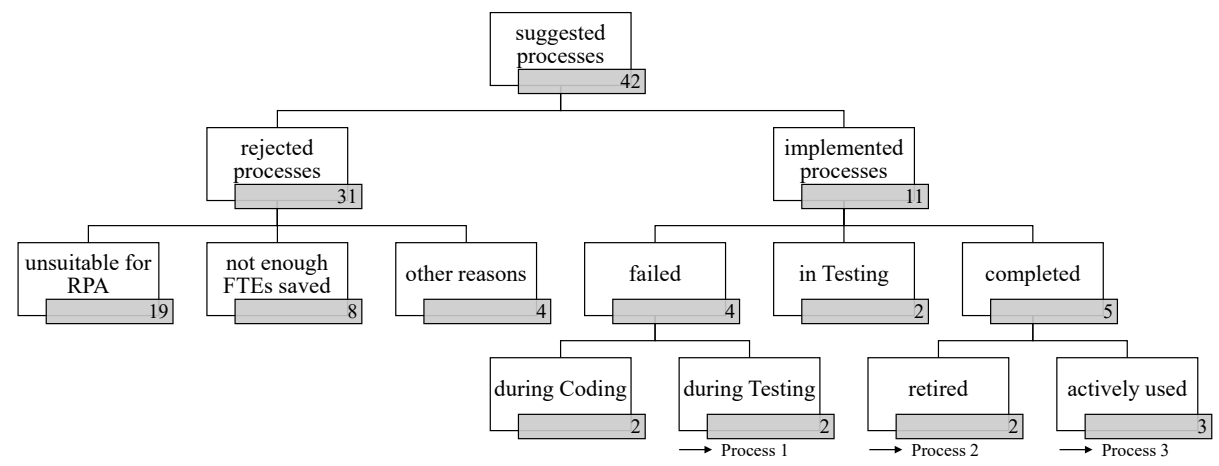

Fig. 1. Processes suggested for automation with RPA.

\subsection{Data Collection Method}

The data is collected from primary and secondary data sources to ensure data triangulation [21]. We conducted semi-structured interviews with the main actors involved in RPA projects. Each interview lasted around one hour. The interviews were recorded and transcribed. The basis for the interview are the general research question, the aspects defined above, and our observations during the RPA projects. The interview guideline is, therefore, structured as follows: 
1. Analysis: process before automation, problems with manual process, and RPA expectations.

2. Product Design: process standardization and process after automation.

3. Coding and Testing: duration and problems.

4. Operation: release date, communication of RPA, problems during usage, effort for maintenance, and lessons learned.

5. Additional comments.

Secondary data originated from (1) the observations we made during process analyses and (2) archival data (e.g., meeting minutes, technical documents, management documents, and reports). In addition, these data were used to validate the statements made during the interviews. Note that the combination of primary and secondary data fosters internal validity of the collected data [21].

\subsection{Data Analysis Method}

All gathered data are analyzed in a structured way to draw conclusions [25] and to answer our research question. First of all, the three cases are treated separately. Problems and challenges are reported for each lifecycle stage and are summarized as lessons learned. Afterwards a cross-case synthesis is performed to generalize the lessons learned from the three cases [21]. The general challenges are derived from the interview results and are presented to the participants for confirmation. After this confirmation, we compare our challenges with findings from the literature to classify our results in current research.

Confidentiality and privacy rules set out by the company are followed all time.

Special attention is paid to validity and reliability of the data analysis [21]. Internal validity is achieved by analyzing data from different data sources. Therefore, eventually biased interview data can be objectified.

\section{Results}

This section describes, analyses, and explains the three selected cases, i.e., Process 1 (Ordering), Process 2 (Construction Report Creation), and Process 3 (Report Generation), separately along the aforementioned lifecycle stages [21].

\subsection{Process 1 - Ordering}

Analysis. Figure 2 visualizes Process 1 before RPA implementation using the Business Process Model and Notation (BPMN). If engineers need a part, they send the order to an employee checking for its completeness. If the order is incomplete, the engineer is notified accordingly to provide the missing details. Otherwise, the employee logs into the order system, copies and pastes the order

details from the e-mail into the system, saves and submits the order, and notifies the engineer about the creation of the order. As problems of this as-is process the 
system is very slow and, therefore, takes a long time to completion. Additionally, the task is a simple copy and paste task. Hence, the management supported the implementation of the bot to relieve the employees from this tedious task.

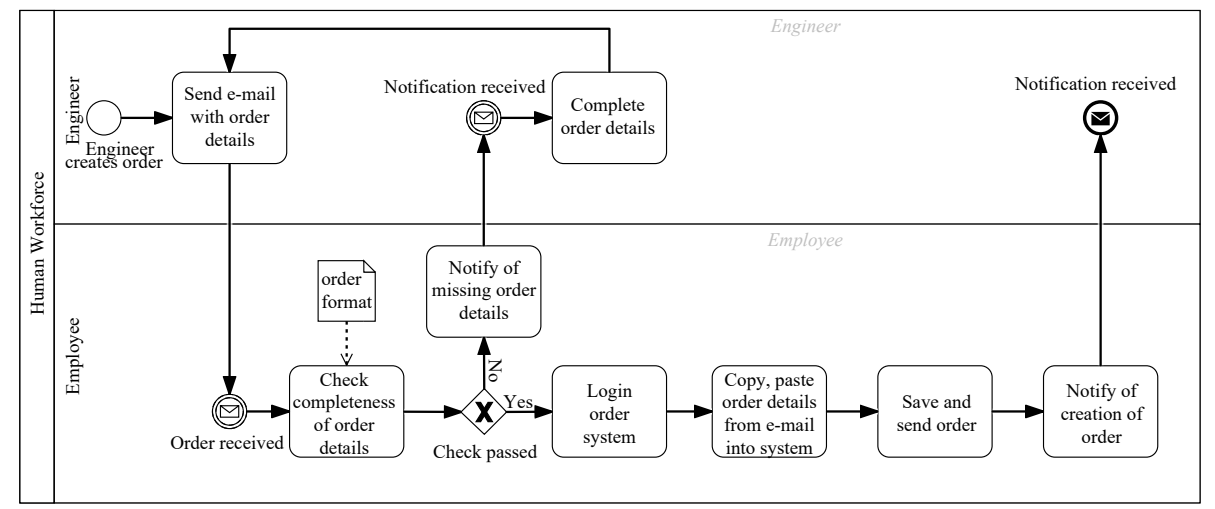

Fig. 2. BPMN Model of Process 1 (Ordering) before RPA implementation.

Product Design. The process has not been standardized prior to RPA implementation. For the engineer the procedure remains the same. For the employee, in turn, an additional check becomes necessary: if the received order is suitable for the RPA bot, it is forwarded to the bot, which then performs the subsequent process steps. Afterwards, the employee is informed, and then needs to submit the order and notify the engineer (cf. Figure 3). If the bot fails, the employee receives a generic error message not providing any details. Therefore, the results from the bot are not the same as if done manually, as one cannot see exactly what error has occurred where.

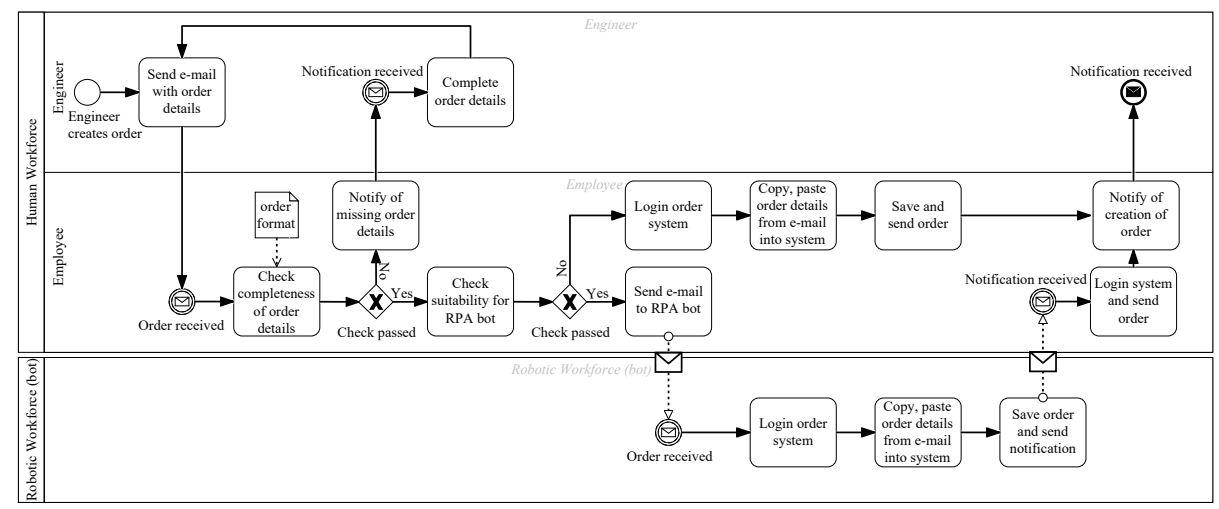

Fig. 3. BPMN Model of Process 1 (Ordering) after RPA implementation. 
Coding/Testing. Coding the RPA bot took the RPA developer 1.5 months, followed by 2 months spent on testing. As major problem during coding, selectors (i.e., attributes of a graphical user interface element to tell the bot how to interact with the interface) did not work properly with the order system and a lot of workarounds with keyboard shortcuts became necessary. Obtaining the necessary access rights for the bot turned out to be difficult. The IT department did not want a bot operating in their order system.

Operation. The operation stage started with problems, as moving the code from integration to production did not work properly. Problems undiscovered in the testing stage occurred, e.g., selectors no longer worked. Employees who should test the bot did not use it after it had turned out that the process differs from the manual one and input documents need to be generated solely for the bot. Therefore, the users preferred staying with the manual process. The RPA developer still spent around 1-2 hours per week for maintaining the bot. In the end, the bot did not go live but was retired after testing due to the sketched problems. In addition, management changed and the new management no longer supported the project.

Lessons Learned. As the most important lesson learned from this RPA project significantly more efforts and time need to be spent in process analysis and documentation. The selected process should be standardized and mature. Furthermore, the process should be precisely documented to facilitate the coding of the RPA bot. Another lesson learned concerns user acceptance: In the given project users did not accept the bot and preferred to execute the process manually.

\subsection{Process 2 - Construction Report Creation}

Analysis. Process 2 describes the generation of reports informing other engineers about changed data (cf. Figure 4). After engineers have corrected construction data, they log into the reporting system to download the report with the newly corrected data. Further, they run an Excel Macro to highlight important data, then save the report on the filesystem, and runs another macro to compare the new version with the previous one. Afterwards, they comment on the changes and inform all concerned engineers accordingly. As a major problem of this process, the reporting system is a legacy system. During the generation of the report, which takes around one hour, the engineers can no longer work on their computers and just have to wait. Additionally, the process is error-prone. The expected savings of using RPA with this process were 2.4 FTEs. Hence, even though management did not support the RPA project and it was clear that the reporting system will soon change, this RPA use case was realized.

Product Design. During product design, the folder structure of the filesystem was standardized. Special attention was paid to design the RPA bot to deliver exactly the same result as if performing the process manually. This resulted in the process depicted in Figure 5: The engineers correct data and log into the reporting system. However, instead of downloading the report right away, they assign the task to the RPA bot. The next day, they can find all prepared 


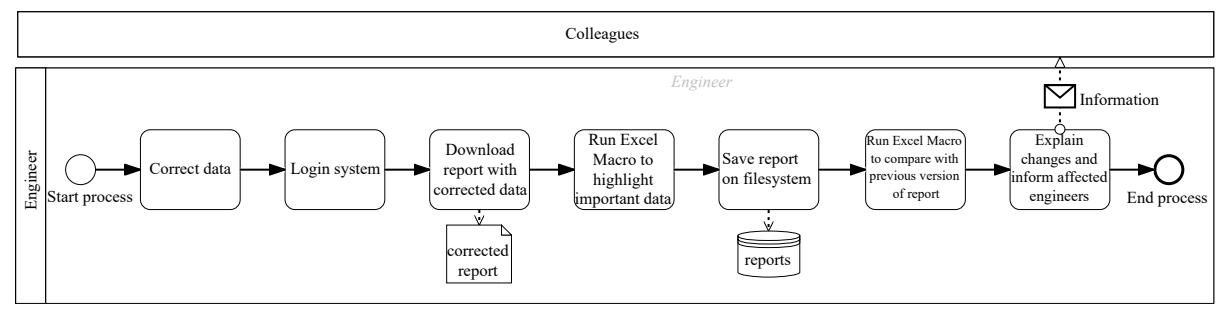

Fig. 4. BPMN Model of Process 2 (Construction Report Creation) before RPA implementation.

documents in the filesystem and focus on commenting and communicating the changes. Twice a day, the RPA bot logs into the reporting system and downloads all assigned reports, runs the Excel Macro to highlight important data, and saves the reports on the filesystem. On the latter, a second Excel Macro is run to compare the new report with its previous version and to save the prepared files on the filesystem. The two processes are decoupled, except for the report database used by both the bot and the engineer.

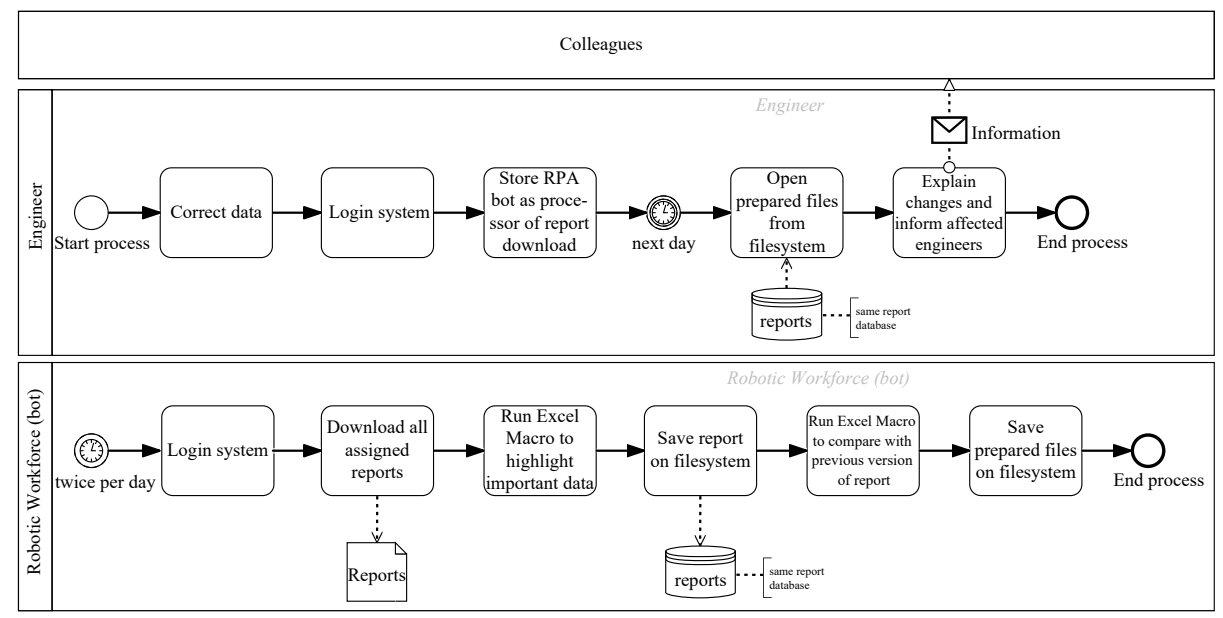

Fig. 5. BPMN Model of Process 2 (Construction Report Creation) after RPA implementation.

Coding/Testing. Coding the RPA bot took the RPA developer 2 weeks, afterwards 2 weeks were spent for testing. No major problems occurred. The bot went live in April 2019. The reporting system was changed one year later, the documents are now generated via server and the bot is no longer needed.

Operation. After going live, the employees who had to use the bot received training, a detailed description of how to use the bot, and a live demonstration. During usage of the bot, no problems occurred for users. Concerning maintenance, the RPA developer spent around one hour per week. 
Lessons Learned. The most important lessons learned from this RPA case are to ensure management support and to standardize inputs. Without support from the management, as in the case of Process 2, it is hard to successfully complete such a project. To ensure the support, it is important to understand what RPA is and what can be done with this technology. According to the RPA developer, most problems with RPA are caused by errors of the employee, e.g., typing errors. Therefore, he suggests only using input from IT tools and design the human bot interaction accordingly. Overall, the RPA developer emphasizes the huge potential for RPA in future, but wishes more support for the RPA developers concerning guidelines and best practices of RPA implementation.

\subsection{Process 3 - Report Generation}

Analysis. In Process 3, reports are generated by logging into the report system and creating the desired specifications (cf. Figure 6). Every Sunday, the engineer receives an automated e-mail from the reporting system. By clicking on the attachment, the report is generated and the engineer can save it on the filesystem. As major problem of this process, the time it takes to generate the report from the attachment is too long. For around 10 minutes the engineer cannot do anything else than waiting for the desired report to be loaded. Therefore, the management supported the RPA implementation of this process.

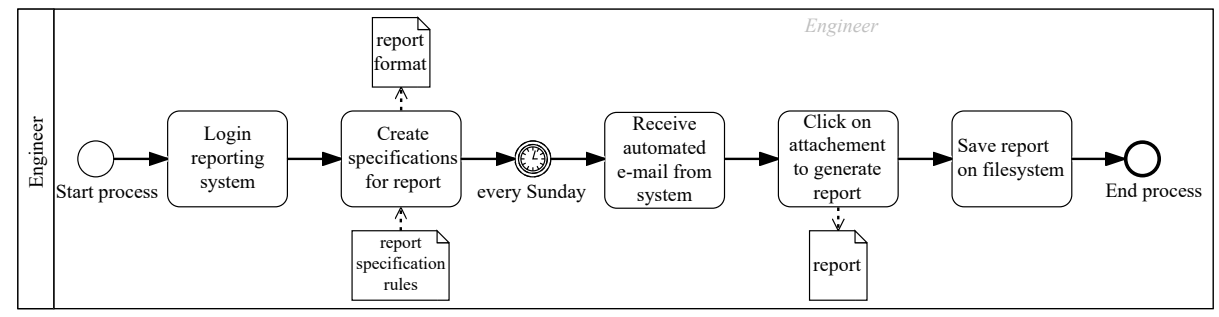

Fig. 6. BPMN Model of Process 3 (Report Generation) before RPA implementation.

Product Design. The product design stage started with standardizing the process and, in particular, the filesystem. The engineers were asked whether a weekly report is sufficiently up-to-date, which they agreed on, especially as the results remain the same as for the manual process. After RPA implementation, the process works as follows (cf. Figure 7): the engineers log into the system, specify the reports they need, and configure the e-mail client such that the bot receives the automated e-mails from the system. For this purpose, every Sunday the bot receives these e-mails, clicks on the attachments, and generates the report. Afterwards, an Excel Macro is executed for comparing the current reports with the one-week old ones and the reports are saved on the filesystem. Consequently, the engineers can always find a weekly up-to-date report on the filesystem. The two workforces work decoupled from one another. However, the 
report is shared among them. If engineers need it, they load it from the filesystem where the bot has saved the report before.

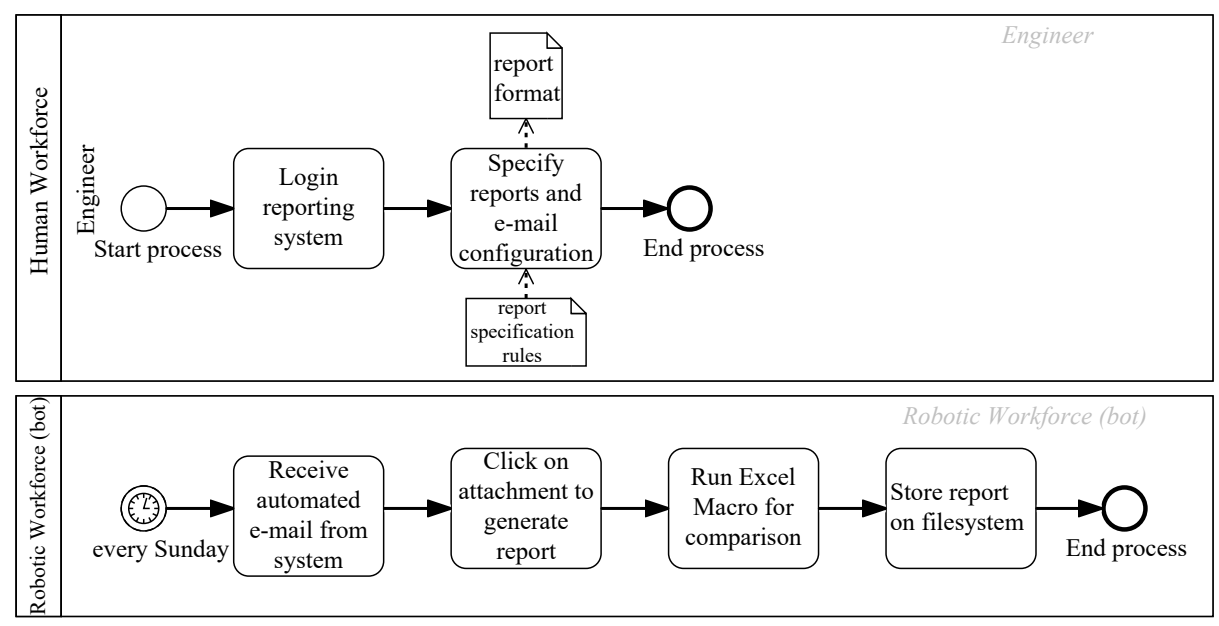

Fig. 7. BPMN Model of Process 3 (Report Generation) after RPA implementation.

Coding/Testing. After two months, the bot was coded and tested by the RPA developer. During this period, no problems occurred. Especially, the bot only required reading access rights for the report system and writing access to the filesystem managed by the department.

Operation. The bot has been live since May 2019 and will be outdated in about 2 years when a new reporting system will be built. Despite the maintenance effort of 2 hours per week, the bot is a great success.

Lessons Learned. Again, the lesson learned in this case is to use standardized input, ideally provided by an IT tool, and to design the human bot interaction accordingly. The developer believes that he has implemented the most appropriate process for RPA. Subsequently proposed processes were not suited for an automation using RPA. Therefore, it is challenging to identify the right process to automate. In general, RPA is difficult to understand for colleagues believing in the automation of everything. The department is quite disillusioned and has no ideas what else could be automated by RPA. Additionally, the developer adds that RPA can still be optimized for maintenance, e.g., to receive information about the bot via e-mail. Finally, the bot developer wishes support in terms of guidelines for RPA development.

\section{Cross-Case Analysis and Derivation of Challenges}

This section discusses the results of all three cases along the bot lifecycle and elaborates on the main challenges of applying RPA in the automotive domain. 
These challenges are compared with the ones reported in RPA literature (cf. Section 2). Finally, threats to validity and limitations of the study are discussed.

\subsection{Cross-Case Analysis}

Analysis. In the analysis stage, the as-is process is analyzed, problems are detected, and goals of the RPA project are recorded. Compared to Processes 2 and 3, Process 1 is rather complex before RPA automation. Processes 2 and 3 are sequential processes without decision points and only one involved actor. All processes shall be automated to save time, which the respective actor would otherwise spend on waiting for the output of different systems. Management support is provided for Processes 1 and 3, whereas this is not the case for Process 2 .

Product Design. During this stage the to-be process is designed and standardized as far as possible. While standardization is possible for Processes 2 and 3, Process 1 cannot be standardized. For Process 1, the to-be process is more complex than the as-is process as an additional check becomes necessary. Still, the results of using the bot differ from the ones of the manual process and are less understandable for the employee. The other two processes require less work than before and the robotic workforce is decoupled from the one of humans. For Processes 2 and 3, special attention is paid to ensure that the results of the bot and the manual process are the same.

Coding/Testing. The coding and testing stage is analyzed with respect to the problems and efforts. Coding and testing are accomplished the fastest for Process 2, after one month the bot is ready and no major issues arise. The implementation of process 3 takes two months with no major problems. Finally, coding and testing of Process 1 takes 3.5 months. Two problems hinder a faster implementation: the selectors do not work properly in the system and getting the access rights granted for the bot is difficult.

Operation. In the operation stage, ongoing maintenance efforts as well as lessons learned are documented. For all three projects maintenance efforts are around 1-2 hours per week.

Lessons Learned. Lessons learned for the three use cases are:

1. Identify the right process to automate and spend sufficient time and efforts into process analysis and documentation. (Processes 1 and 3)

2. Understand the factors influencing user acceptance of RPA. (Process 1)

3. Explain RPA and the benefits of this technology. (Processes 2 and 3)

4. Properly design the interaction between human and RPA bot. (Processes 2 and 3)

5. Provide software development guidelines for RPA. (Processes 2 and 3)

Table 1 summarizes our main findings and compares the three cases.

\subsection{Derivation of Challenges}

The five lessons learned are compared with findings from literature. Further, we highlight future research possibilities to overcome the challenges. 
Table 1. Overview of the three use cases.

\begin{tabular}{|c|c|c|c|c|}
\hline $\begin{array}{l}\text { Lifecycle } \\
\text { stage }\end{array}$ & Aspect & Process 1 & Process 2 & Process 3 \\
\hline \multirow[t]{3}{*}{ Analysis } & As-is process & $\begin{array}{l}\text { complex, decision } \\
\text { points, multiple ac- } \\
\text { tors involved }\end{array}$ & $\begin{array}{l}\text { linear, one actor in- } \\
\text { volved }\end{array}$ & $\begin{array}{l}\text { linear, one actor in- } \\
\text { volved }\end{array}$ \\
\hline & $\begin{array}{l}\text { Reason for au- } \\
\text { tomation }\end{array}$ & time savings & $\begin{array}{l}\text { time savings, error } \\
\text { reductions }\end{array}$ & time savings \\
\hline & $\begin{array}{l}\text { Management } \\
\text { support }\end{array}$ & yes & no & yes \\
\hline \multirow{4}{*}{$\begin{array}{l}\text { Product } \\
\text { Design }\end{array}$} & $\begin{array}{l}\text { Standard- } \\
\text { ization }\end{array}$ & no & yes & yes \\
\hline & To-be process & $\begin{array}{l}\text { additional check, } \\
\text { more complex }\end{array}$ & less work & less work \\
\hline & $\begin{array}{l}\text { Human-Bot- } \\
\text { Interaction }\end{array}$ & $\begin{array}{l}\text { interaction via e- } \\
\text { mail and user input }\end{array}$ & $\begin{array}{l}\text { user assigns task to } \\
\text { bot, gets result the } \\
\text { next day }\end{array}$ & $\begin{array}{l}\text { specify once the re- } \\
\text { port and receive } \\
\text { it up-to-date every } \\
\text { week }\end{array}$ \\
\hline & $\begin{array}{l}\text { Manual vs. } \\
\text { Bot Result }\end{array}$ & different & same & same \\
\hline \multirow{2}{*}{$\begin{array}{l}\text { Coding/ } \\
\text { Testing }\end{array}$} & Time effort & 3.5 months & 1 month & 2 months \\
\hline & Problems & $\begin{array}{l}\text { with selectors and } \\
\text { access right for bot }\end{array}$ & no & no \\
\hline \multirow[t]{2}{*}{$\begin{array}{l}\text { Opera- } \\
\text { tion }\end{array}$} & $\begin{array}{l}\text { Maintenance } \\
\text { effort }\end{array}$ & $1-2 \mathrm{~h} /$ week & $1 \mathrm{~h} /$ week & $2 \mathrm{~h} /$ week \\
\hline & $\begin{array}{l}\text { Lessons } \\
\text { Learned }\end{array}$ & $\begin{array}{l}\text { identify the right } \\
\text { processes to auto- } \\
\text { mate, understand } \\
\text { factors influencing } \\
\text { user acceptance }\end{array}$ & $\begin{array}{l}\text { design the human } \\
\text { bot interaction, } \\
\text { explain RPA and } \\
\text { its merits, provide } \\
\text { software develop- } \\
\text { ment guidelines }\end{array}$ & $\begin{array}{l}\text { design the human } \\
\text { bot interaction, } \\
\text { explain RPA and } \\
\text { its merits, provide } \\
\text { software develop- } \\
\text { ment guidelines, } \\
\text { identify the right } \\
\text { process to auto- } \\
\text { mate, }\end{array}$ \\
\hline \multicolumn{2}{|c|}{ Evaluation } & $\begin{array}{l}\text { failed before } \\
\text { live, money } \\
\text { FTEs spent }\end{array}$ & $\begin{array}{l}2.4 \text { FTEs saved, } 13 \\
\text { months of usage }\end{array}$ & $\begin{array}{l}1.2 \text { FTEs saved, } \\
\text { used for } 15 \text { months, } \\
\text { will be used for } 2 \\
\text { years }\end{array}$ \\
\hline
\end{tabular}

The challenge to identify the right processes for automation is addressed in $[5,8,10]$. [5] emphasizes that the processes need to be carefully selected and recommends the choice of rule-based processes, which usually require a lot of time and resources. [8] suggests selecting standardized and stabilized processes as candidates for RPA. [10] points out that RPA is one process automation approach among others (e.g., screen scraping) and, therefore, processes for RPA need to be chosen with care. However, to the best of our knowledge, there is no comprehensive overview of existing process selection methods. Apart from an overview of these methods, an assessment regarding applicability in the automotive domain is desirable. Future research should, therefore, focus on providing an overview and assessment of process selection methods for RPA.

The challenge to understand the factors influencing RPA user acceptance is covered partly in $[5,8,18]$. [5] suggests addressing concerns about job losses early on such that the employees are more comfortable using the 
software bots. Similarly, [18] encounters the challenge of convincing employees to change their work habit. [8] indicates that a culture of business innovation and technology fosters RPA adoption. Nonetheless, these are only some aspects, which probably influence user acceptance of RPA. A more general approach that covers the variety of factors influencing RPA user acceptance needs to be developed. Based on the identified factors, further RPA software improvements can be achieved.

How to explain RPA and its merits is discussed in $[10,12,16]$. [10] suggests preparing the employees accordingly, whereas [16] emphasizes the need to manage the impact of RPA carefully. In any case, a transparent communication with the concerned employees becomes necessary from the beginning. In [12], the same challenge is discussed, namely, the lack of RPA understanding, with the recommendation to provide background knowledge to the employees. Future work should, therefore, provide new ways of explaining RPA to employees. To the best of our knowledge, there are no sophisticated communication concepts for explaining RPA and its advantages to the concerned employees. Special attention needs to be paid to adapt these concepts to highly skilled engineers.

The challenge to design the interactions between the employee and the bot could be linked to user acceptance, e.g., the interactions should be designed in a way such that the change of work habit becomes minimal [18]. [23] states that robots need more explicit instructions than humans. This is one aspect to be considered in the interaction design. However, many more aspects should be considered to provide guidance for a good user interface design in RPA. Future work should develop an evaluation model to assess the interaction between employee and RPA bot. The results could then be used to derive recommendations for the user interface design resolving the discovered challenge.

Finally, the challenge of how to implement RPA solutions has been tackled by only few works. [9] states that components should be reused to enable faster bot implementation, [16] emphasizes the need for an agile RPA development. Details, models or recommendation for an RPA development constitute another aspect of future work. Finally, the software development guidelines should be tailored to the requirements of an engineer as well.

In a nutshell, the challenges we discovered in the automotive domain have been at least partly addressed in the literature that deals with RPA implementations in other domains.

\subsection{Threats to Validity and Limitations}

With the presented exploratory case study, we gathered experiences with RPA projects in a real environment. However, there are several threats to validity and limitations of our research.

As a first limitation, the case study is solely conducted in one domain, i.e., automotive engineering, covering three different projects. Nevertheless, we believe that the obtained results are generalizable to other domains and projects for the following two reasons: 1) In the literature, we can find RPA case studies in different areas deriving similar challenges and 2) we have insights into additional 
RPA projects from the automotive industry (cf. Figure 1), which confirm this. Unfortunately, we cannot provide more information on these additional studies due to confidentiality reasons. To ensure external validity and confirm the generalization of our results, new case studies in other domains and companies need to be conducted as well.

Second, one may argue that the discovered challenges are not RPA-specific, but might be observed in other software projects as well. We briefly sketch differences between RPA and traditional software projects [15]: The goal of RPA is to create bots that run independently and human only hand over the task. Contrariwise, traditional software is designed to support the human in executing tasks. Further, RPA bots are mostly implemented by domain experts [22], whereas the implementation of traditional software is done by software engineers from the IT department. Therefore, RPA projects have to be addressed in another way than traditional software projects.

\section{Summary and Outlook}

The presented exploratory case study provides insights into concrete RPA projects from the automotive industry. The discovered challenges, i.e., to identify the appropriate processes for RPA, to understand the factors influencing user acceptance, to explain RPA to employees, to design human bot interaction, and to provide RPA software development guidelines, will be further addressed by us to ensure a successful application of RPA in practice.

To be more precise, we are working on a framework addressing these challenges holistically. For example, the challenge to understand the factors influencing user acceptance is addressed in [19], which develops a model for assessing RPA user acceptance as well as the factors influencing it. These factors are used to derive recommendations for designing and implementing RPA bots with increased user acceptance among employees using the bots in their daily work.

Further case studies and studies are necessary to enhance these results, confirm findings, and ensure external validity. The same case study method should be used by further research to discuss RPA use cases and to generate comparable results.

\section{References}

1. Aguirre, S., Rodriguez, A.: Automation of a Business Process Using Robotic Process Automation (RPA): A Case Study. In: Workshop on Eng Appl. pp. 65-71. Springer (2017)

2. Asatiani, A., Penttinen, E.: Turning robotic process automation into commercial success - Case OpusCapita. J Inf Technol Teach Cases 6(2), 67-74 (2016)

3. Asquith, A., Horsman, G.: Let the robots do it! - Taking a look at Robotic Process Automation and its potential application in digital forensics. Forensic Sci Int Reports 1 (2019) 
4. Chacón-Montero, J., Jiménez-Ramírez, A., Enríquez, J.G.: Towards a Method for Automated Testing in Robotic Process Automation Projects. In: 14th Int Workshop on Automation Softw Test. pp. 42-47. IEEE Press (2019)

5. Hallikainen, P., Bekkhus, R., Pan, S.L.: How OpusCapita Used Internal RPA Capabilities to Offer Services to Clients. MIS Q Exec 17(1), 41-52 (2018)

6. Ivančić, L., Vugec, D.S., Vukšić, V.B.: Robotic Process Automation : Systematic Literature Review. In: Int Conf Bus Process Manag, vol. 361, pp. 280-295 (2019)

7. Jiménez-Ramírez, A., Chacón-Montero, J., Wojdynsky, T., González Enríquez, J.: Automated testing in robotic process automation projects. J Softw Evol Process pp. 1-11 (2020)

8. Lacity, M., Willcocks, L., Craig, A.: Robotic Process Automation at Xchanging. Outsourcing Unit Work Res Pap Ser 15(3), 1-26 (2015)

9. Lacity, M., Willcocks, L., Craig, A.: Robotic Process Automation: Mature Capabilities in the Energy Sector. Outsourcing Unit Work Pap Ser pp. 1-19 (2015)

10. Lacity, M., Willcocks, L., Craig, A.: Robotizing Global Financial Shared Services at Royal DSM. Outsourcing Unit Work Res Pap Ser 16(2), 1-26 (2016)

11. Lohrmann, M., Reichert, M.: Effective application of process improvement patterns to business processes. Softw \& Systs Model 15(2), 353-375 (2016)

12. Radke, A.M., Dang, M.T., Tan, A.: Using Robotic Process Automation (RPA) to enhance item master data maintenance process. LogForum 16(1), 129-140 (2020)

13. Royce, W.W.: Managing the development of large software systems: concepts and techniques. In: 9th Int Conf Softw Eng. pp. 328-338 (1987)

14. Runeson, P., Höst, M., Rainer, A., Regnell, B.: Case Study Research in Software Engineering: Guidelines and Examples (2012)

15. Rutschi, C., Dibbern, J.: Towards a framework of implementing software robots: Transforming Human-executed Routines into Machines. Data Base Adv Inform Syst 51(1), 104-128 (2020)

16. Schmitz, M., Dietze, C., Czarnecki, C.: Enabling digital transformation through robotic process automation at Deutsche Telekom. In: Urbach, N., Röglinger, M. (eds.) Digitalization Cases, Management for Professionals, pp. 15-33 (2019)

17. Syed, R., Suriadi, S., Adams, M., Bandara, W., Leemans, S.J., Ouyang, C., ter Hofstede, A.H., van de Weerd, I., Wynn, M.T., Reijers, H.A.: Robotic Process Automation: Contemporary Themes and Challenges. Comput Ind 115 (2020)

18. Viale, L., Zouari, D.: Impact of digitalization on procurement: the case of robotic process automation. Supply Chain Forum An Int J pp. 1-11 (2020)

19. Wewerka, J., Dax, S., Reichert, M.: A User Acceptance Model for Robotic Process Automation. In: IEEE Int Ent Dist Obj Comp Conf (2020)

20. Wewerka, J., Reichert, M.: Towards Quantifying the Effects of Robotic Process Automation. In: IEEE Int Ent Dist Obj Comp Conf Workshop on Front Process Aware Sys (2020)

21. Wieringa, R.J.: Design science methodology for information systems and software engineering. Springer (2014)

22. Willcocks, L., Lacity, M.: Robotic Process Automation: The Next Transformation Lever for Shared Services. Outsourcing Unit Work Res Pap Ser 15(7), 1-35 (2015)

23. Willcocks, L., Lacity, M.: Robotic Process Automation at Telefónica O2. MIS Q Exec 15(1), 21-35 (2016)

24. William, W., William, L.: Improving Corporate Secretary Productivity using Robotic Process Automation. In: Int Conf Technol Appl Artif Intell. pp. 1-5 (2019)

25. Yin, R.K., et al.: Case Study Research: Design and methods (2003) 